\title{
Evaluating the use of corticosteroids in preventing and treating bronchopulmonary dysplasia in preterm neonates
}

This article was published in the following Dove Press journal: International Journal of General Medicine

\author{
Oreoluwa Olaloko' \\ Raihan Mohammed' \\ Utkarsh Ojha ${ }^{2}$ \\ 'Faculty of Medicine, University of \\ Cambridge, Cambridge, UK; ${ }^{2}$ Faculty \\ of Medicine, Imperial College, London, \\ UK
}

\begin{abstract}
Approximately 15 million babies worldwide are born premature, and complications of prematurity are one of the leading causes of death in neonates. Neonatal respiratory distress syndrome (NRDS) and bronchopulmonary dysplasia (BPD) are two of the most common and serious consequences of prematurity. Synthetic corticosteroids, including dexamethasone, have been central in efforts to treat and prevent BPD. There is strong evidence to show that prenatal corticosteroids reduce infant mortality and the incidence of NRDS, leading to their widespread use in obstetric units. However, data suggest that they are not as effective in reducing the incidence of BPD as NRDS, which may be due to the multifactorial pathogenesis of BPD. On the other hand, the use of postnatal corticosteroids in preterm infants is much more controversial. They have been shown to improve lung function and help in reducing the need for mechanical ventilation. These benefits, however, are associated with a range of adverse short- and long-term effects. This review will discuss the benefits and consequences of corticosteroids in treating BPD and will examine alternative treatments and future research that may improve the understanding of BPD and inform clinical practice.
\end{abstract}

Keywords: bronchopulmonary dysplasia, corticosteroids, preterm birth, prenatal, postnatal

\section{Introduction}

Preterm birth is defined as birth that occurs before 37 weeks gestational age. Worldwide, $\sim 15$ million babies were born preterm in 2010 and the complications of preterm birth are a significant cause of death of neonates. Annually, $35 \%$ of the 3.1 million neonatal deaths are estimated to be caused by the complications of preterm birth. ${ }^{1}$ These causes of prematurity are often unknown, but there are maternal risk factors, such as a history of preterm birth and short cervical length, which increase the risk. ${ }^{2}$

One of the most common morbidities in premature infants is neonatal respiratory distress syndrome (NRDS). NRDS is most often a consequence of lung immaturity, which results in an underdeveloped structure and a deficiency of important compounds, especially surfactant. ${ }^{3}$ Surfactant deficiency in the lungs increases the risk of lung collapse, resulting in difficulty breathing for the infant. NRDS is a significant cause of mortality in preterm neonates, especially very preterm neonates born at $<30$ weeks. ${ }^{4}$ Standard treatment for NRDS includes surfactant replacement therapy, breathing aided by mechanical ventilation via an endotracheal tube or continuous positive airway pressure applied via the nasopharynx. Surfactant replacement is on the World Health Organization model list of essential medicines and reduces mortality in preterm infants on mechanical ventilation. ${ }^{5}$ However, despite the improvements in neonatal intensive
Correspondence: Raihan Mohammed Faculty of Medicine, Robinson College, University of Cambridge, Grange Road, Cambridge CB3 9AN, UK

Tel +44787 I736004

Emailrm758@cam.ac.uk 
care in treating NRDS, bronchopulmonary dysplasia (BPD), or chronic lung disease, is still an important consequence of NRDS.

Historically, definitions of BPD have lacked uniformity and have developed over time. The earliest clinical description was by Northway et $\mathrm{al}^{6}$ who described it as a condition when an infant has persistent respiratory signs and symptoms, requires supplemental oxygen at 28 days of age to maintain $\mathrm{PaO}_{2}$ above $50 \mathrm{mmHg}$ and has abnormal chest radiographs. Later, Bancalari et al modified this original definition by adding that infants should also have received mechanical ventilation for at least 3 days in the first postnatal week ${ }^{7}$ and Shennan et al determined that treatment with supplemental oxygen at 36 weeks' postmenstrual age (PMA) better predicted pulmonary outcomes. ${ }^{8}$ However, these definitions lacked specificity, and failed to account for important clinical distinctions related to extremes of prematurity and the wide variability in criteria for using prolonged oxygen therapy. Now, the definition includes total duration of oxygen supplementation, positive pressure requirements and gestational age, in addition to oxygen dependency at 36 weeks PMA. ${ }^{9}$ This approach at defining BPD categorizes its severity (which influences pulmonary and neurodevelopmental outcomes) and defines the increasing risk of mortality along a spectrum.

The prolonged delivery of high levels of oxygen can cause alveolar septal injury, bronchiole inflammation and pulmonary vascular remodeling. ${ }^{10}$ Thus, consequences of BPD can include difficulty in breathing and hypoxemia, and $\sim 40 \%$ develop pulmonary hypertension, ${ }^{11}$ which may be fatal. BPD also worsens the adverse neurodevelopmental outcomes such as behavioural impairments that are associated with very preterm neonates. ${ }^{12}$ The first descriptions of the negative effects of mechanical ventilation resulting in BPD were made in observations in $1967 .{ }^{6}$ It results from damage and inflammation to developmentally immature lungs. ${ }^{13}$ Although mechanical ventilation in the treatment of NRDS is often the cause of this damage, BPD is a multifactorial disorder and also occurs in infants who do not receive mechanical ventilation.

This review will discuss the use of corticosteroids in the treatment and prevention of BPD. We begin with a brief discussion on the use of prenatal corticosteroids, which are used to help accelerate maturation of fetal organ systems and reduce the incidence of many complications of prematurity. We then debate the use of postnatal corticosteroids to prevent and treat BPD, which is much more controversial than their use prenatally, as well as any alternative treatments. Finally, we conclude with a discussion on the future directions for research that may help further our understanding of BPD and may be used to inform clinical practice.

\section{Fetal maturation and corticosteroids}

Corticosteroids are a type of steroid hormone produced by the zona glomerulosa and fasciculata of the adrenal cortex. The term corticosteroid also refers to the synthetic analogs of the organic hormones, such as betamethasone. Corticosteroids can be divided into glucocorticoids and mineralocorticoids, which are involved in a wide range of physiological processes including inflammation, metabolism, immunity and electrolyte regulation. The actions of corticosteroids relevant to BPD are their role in the maturation of fetal organs during gestation and their anti-inflammatory actions. A common corticosteroid used in perinatal care is dexamethasone, an artificial corticosteroid which can be administered intravitreally, intramuscularly, intravenously and orally.

Many of the complications of preterm birth are a consequence of the immaturity of various organ systems. The transition to life independent of the mother is a significant and vulnerable period during development and several developmental changes in the fetus must occur during gestation to prepare for this transition. For example, changes occur in the newborn's body temperature, blood flow resistance and liver enzymes. In particular, the pulmonary system must undergo drastic changes as the fetus switches from carrying out gaseous exchange at the placenta to the lungs. From the first breath within about 10 seconds after delivery, the lungs begin to inflate, and fluid is drained or absorbed from the respiratory system. Several studies have indicated the importance of the endogenous glucocorticoid cortisol in regulating the maturation of fetal organs and systems during gestation. ${ }^{14}$ Corticosteroids administered prenatally have been shown to improve cardiovascular function; fetal cortisol infusion increased blood pressure in sheep by $5-10 \mathrm{mmHg}$ and decreased fetal heart rate variability. ${ }^{15}$ Furthermore, prenatal dexamethasone has been shown to improve respiratory function by stimulating surfactant production in the lungs of fetal rats. ${ }^{16}$ The main lipid component of surfactant, dipalmitoylphosphatidylcholine, reduces the surface tension in the alveoli of the lungs by adsorbing to the air-water interface of alveoli. This helps to prevent collapse of the lungs after birth when the infant must breathe independently. ${ }^{17}$ Thus, premature birth interrupts the maturation of the fetus, resulting in postnatal complications such as BPD and NRDS. This has led to the rationale of administering prenatal synthetic corticosteroids to improve outcomes in pregnant women at risk of preterm labor and birth. This facilitates a smoother 
adaptation to postnatal life for the fetus and reduces the incidence of BPD and NRDS.

\section{Prenatal corticosteroids Benefits of prenatal corticosteroids}

Administration of prenatal corticosteroids aims to reduce the incidence of complications and morbidities associated with preterm birth, including NRDS and BPD. Maternal administration of corticosteroids prior to preterm birth causes thinning of the primary septa. This narrows the air blood barrier, stimulates the production of surfactant, which stabilizes the alveolar sacs and prevents collapse after exhalation, and stimulates the clearance of fetal lung fluid. ${ }^{18}$ The first randomized controlled trial (RCT) of betamethasone in humans was conducted by Liggins and Howie in $1972 .{ }^{19}$ The trial was carried out in 282 women threatened with premature delivery and showed strong evidence for the effectiveness of prenatal corticosteroids in reducing neonatal complications and mortality. The incidence of NRDS in the betamethasone-treated group was $9.0 \%$, while in the control group the incidence was $25.8 \%$. Furthermore, early infant mortality was $3.2 \%$ in the betamethasone-treated group and $15.0 \%$ in the control group.

Since that seminal study, several more trials have been conducted which further support the positive results in reducing infant mortality and complications of prematurity. A Cochrane review of 30 trials found that a single course of antenatal corticosteroid given to the mother reduced the incidence of several complications of NRDS, such as intraventricular hemorrhage and necrotizing enterocolitis, as well as the rates of infant mortality. ${ }^{3}$ The success of prenatal corticosteroids in reducing the complications of preterm birth has led to its widespread adoption across neonatal intensive care units (NICUs) in developed countries.

\section{Adverse effects of prenatal corticosteroids}

Despite the effectiveness of prenatal synthetic corticosteroids in reducing morbidity, such as NRDS, and mortality in preterm neonates, the rate of BPD in these infants has not decreased. Although the rate of death from BPD has decreased, the disease is not prevented, and this may be due to increased survival of very immature infants at high risk of $\mathrm{BPD}^{20}$ or its multifactorial pathogenesis. One study has shown that BPD incidence was reduced when prenatal steroids were administered; however, this was a retrospective study conducted with only those women who had premature rupture of membranes and found no benefit with regard to the incidence of intraventricular hemorrhage or neonatal hospitalization. ${ }^{21}$
In addition to this, there are also concerns about some of the adverse effects that the treatment may cause. Corticosteroids have several targets in cells across the body leading to a variety of systemic effects, both beneficial and undesirable. Single course administration has been shown to be largely safe, but some studies have reported reduction in fetal body movements, fetal breathing movements and heart rate variation after betamethasone administration. ${ }^{22}$ Furthermore, in a randomized trial of single versus serial courses of antenatal corticosteroids, a reduction in birth weight and an increase in the number of infants who were small for gestational age were found, especially after four courses of corticosteroids. ${ }^{23}$ Some studies on rats have suggested a link between fetal conditions and adult disease, which is known as developmental programming. Exposure to excess glucocorticoids as a fetus is associated with low birth weight and cardiovascular disease in adult life due to activation of the hypothalamic-pituitary-adrenal axis. ${ }^{24}$ However, there is no consensus yet on how the use of antenatal steroids can be optimized by improving the timing of administration and dosing. ${ }^{25}$

Overall, there is strong evidence to show that the use of prenatal corticosteroid treatment in women who are threatened with a potential preterm delivery reduces the risk of complications and infant mortality. Due to the relative ineffectiveness of prenatal corticosteroids in reducing the rates of BPD, however, alternative treatments should be investigated to prevent and treat this lung condition. Postnatal corticosteroids, therefore, may be an option to treat BPD if complications arise after premature birth.

\section{Inflammation and corticosteroids}

The pathogenesis of BPD is multifactorial and has not been fully elucidated. There are a number of factors known to contribute to its development. For example, barotrauma, or volutrauma, may have an important role in the etiology of BPD in many cases. ${ }^{20}$ Barotrauma refers to damage caused by changes in air pressure, which can occur from mechanical ventilation (positive pressure ventilation) via the endotracheal tube. Other risk factors may play a part in the development of BPD, including infection and a patent ductus arteriosus (PDA). A persistent left-right shunt through a PDA increases the rate of hydrostatic fluid filtration into the lung's interstitium, impairs pulmonary mechanics and prolongs the need for mechanical ventilation. ${ }^{26}$ Thus, pharmacological PDA closure should lead to improved postnatal alveolar development; however, there is little evidence to support or refute a causal role of PDA in the development of BPD. In fact, long-term outcomes of PDA closure demonstrate no 
pulmonary benefit and may even have increased incidence of BPD due to arrest in alveolarization in the lungs. ${ }^{27}$ Persistent alterations in inflammatory mediators and epithelial sodium channels $(\mathrm{ENaC})$ may also account for the lack of improvement in pulmonary mechanics and BPD following surgical closure. This inflammation may also be in response to respiratory infection. Animal models in lambs and transgenic mice have shown that inflammatory mediators such as tumor necrosis factor and transforming growth factor beta affect alveolar septation and increase the risk of BPD. ${ }^{13}$ Although the direct role of infection in the development of BPD is not completely understood, evidence suggests that systemic inflammation, associated with vascular permeability alterations, is a major cause of cell injury and the consequent alveolarization process interruption. Finally, there may also be genetic factors involved in determining the risk of BPD. Genetic association studies have primarily focused on the components of innate (e.g., first-line) immune and antioxidant defenses, mechanisms of vascular and lung remodeling, and surfactant proteins. However, studies have been limited in sample size and, therefore, fraught with a high probability of false-positive and -negative associations.

Given that inflammation appears to be important in the pathogenesis of BPD, anti-inflammatory agents (such as corticosteroids) would be an obvious option for potential treatments. However, the mechanism of action of corticosteroids in the treatment of BPD is not fully known. One suggestion is through the inhibitory actions of proinflammatory mediators. Corticosteroids have been demonstrated to inhibit the release of the proinflammatory arachidonic acid by over $50 \%$ from cellular lipids of in vitro mouse fibroblasts. ${ }^{28}$ Arachidonic acid is metabolized to a wide range of proinflammatory eicosanoids, causing vasodilation, neutrophil activation and hyperalgesia. A study on dexamethasone treatment in preterm infants at risk of BPD found that it reduced the number of neutrophils in the lung aspirate fluid and decreased the concentrations of leukotriene, another proinflammatory mediator, when given 10 days after birth. ${ }^{29}$ Hence, decreasing inflammatory responses in the lungs may help to reduce the incidence or severity of BPD. However, use of corticosteroids in this way is controversial, as there is ongoing discussion on whether the benefits they provide outweigh the systemic negative effects.

\section{Postnatal corticosteroids}

\section{Benefits of postnatal corticosteroids}

An early trial using dexamethasone found that lung function in infants with BPD was improved. ${ }^{30}$ In this RCT, dexamethasone was administered to six premature infants with BPD at an equivalent gestational age of 27-33 weeks. Respiratory rate and peak inspiratory pressure were some of the measurements used as indicators of lung function. The authors found that dexamethasone improved the lung function and reduced the need for mechanical ventilation, facilitating extubation. Their results suggested that dexamethasone may be beneficial in the treatment of BPD, particularly if it is a result of damage due to mechanical ventilation. Improved lung function and earlier removal from mechanical ventilation appear to be some of the main short-term benefits of postnatal corticosteroids in the treatment of BPD. However, there were only six subjects in the study and they were at a much later gestational age than the current BPD patients.

More recent trials confirmed the results of this initial RCT, demonstrating associations between postnatal corticosteroids and improved lung function and reduced dependency on mechanical ventilation. ${ }^{31}$ Early or prophylactic use of low-dose hydrocortisone in infants $<28$ weeks was shown to reduce risk of BPD. ${ }^{32}$ These short-term benefits of corticosteroids in the treatment of BPD led to its increased use in preterm infants. A retrospective analysis of neonatal care networks in the USA and Canada found that postnatal corticosteroid use increased during the early 1990 s to a peak in $1998 .{ }^{33}$ However, as the use of corticosteroids grew, so did concern over its adverse short-term effects. Rates of postnatal corticosteroid use declined from $20 \%$ in 1997-2000 to only $12 \%$ in 2001-2002 and then again to $8 \%$ in 2004 to the present rate. ${ }^{34}$ The question of whether the benefits outweighed the accompanying adverse effects became increasingly important.

\section{Adverse effects of postnatal corticosteroids}

A Cochrane systematic review of 32 RCTs on the effects of postnatal corticosteroids administered within the first week after birth similarly found that corticosteroids had beneficial short-term effects in improving the lung function and reducing dependency on mechanical ventilation, but they were coupled with adverse side effects. ${ }^{35}$ The review established that there was decreased risk of BPD at 28 days and that corticosteroid treatment facilitated extubation. Long-term follow-up studies report increased risk of abnormal findings on neurologic examination and increased risk of cerebral palsy (CP). Adverse neurologic outcomes were reported in children given a short (4 or 6 week) tapering course of dexamethasone in several studies; data showed about a threefold increased risk of CP among survivors, including children with spastic diplegia, spastic quadriplegia and hemiplegia. 
Systemic postnatal corticosteroid therapy to treat BPD has been implicated in increasing $\mathrm{CP}$ risk, ${ }^{36}$ although in many of these studies, $\mathrm{CP}$ was diagnosed with certainty before 5 years of age, which is problematic. Furthermore, these studies had a low follow-up rate; only Yeh (1997) had a 92\% follow-up and the results had no significant difference in $\mathrm{CP}$ at $8-9$ years - an age when diagnosing $\mathrm{CP}$ is more certain. ${ }^{37}$

Many studies in the review reported a range of negative side effects associated with the treatment. Adverse short-term side effects found in many of the trials included gastrointestinal bleeding, intestinal perforation, hyperglycemia, hypertension, hypertrophic cardiomyopathy and growth failure. Importantly, there was also no significant improvement in the rate of neonatal mortality despite the benefits seen. Furthermore, cardiovascular problems, such as thickening of interventricular septum and a decrease in size of the left ventricle, have been reported in preterm infants treated with intravenous dexamethasone for 7 days. ${ }^{38}$

Due to these risks, the authors concluded that the benefits of postnatal corticosteroid treatment do not outweigh the negative consequences. However, the authors' review did not include trials of some alternative methods of administration that are sometimes used, such as inhaled corticosteroids. This omission of alternative methods of administration, and the different dosing regimens and preparations used may limit the clinical applicability of these conclusions. This was addressed in a further Cochrane systematic review which demonstrated that early use of inhaled steroids was not associated with any significant difference in the incidence of death or BPD at 36 weeks PMA or at 28 days of age compared with the early use of systemic steroids. ${ }^{39}$

In addition, an RCT of 248 infants reported that postnatal dexamethasone, administered within 3 days of birth, reduced the need for mechanical ventilation at 3 days. ${ }^{40}$ This difference did not last, as by 7 days, there was no difference in the need for mechanical ventilation in the dexamethasone and placebo groups. Furthermore, there was no significant difference in survival between the dexamethasone group and the placebo group. There was also no significant difference in the incidence of BPD between the two groups. These results would indicate against the use of early dexamethasone in the prevention of BPD or in the reduction of mortality.

Glucocorticoids bind to their receptor, which is almost ubiquitously expressed. Thus, they affect virtually all immune cells and have a systemic immunosuppressant effect by suppressing cell-mediated immunity (e.g., interleukins, tumor necrosis factor-alpha). In the early trial on dexamethasone, ${ }^{30}$ three of the six patients in the trial survived. Five of the six patients had infections while they were treated with dexamethasone and two of the deaths were due to pneumonia. It is important, however, to consider that the patients selected for this study already had a poor prognosis before the trial, and thus, this may have affected the results.

Newborn infants, especially very low birth weight and preterm infants, already have a high susceptibility to infections. It is difficult to fully determine what proportion of the complications and deaths due to infection in these studies are a consequence of the immunosuppressive actions of corticosteroids, or as a consequence of the immature neonatal immune system. For example, although there are some trials that report increased rates of infection, others using dexamethasone observed no significant difference in infection rates compared with the control group. ${ }^{41}$ These may also be a result of differences in experimental procedure and practices across different NICUs. Furthermore, these studies suffer from selection bias, as many of the infants involved are of very low birth weight and, therefore, have a poorer prognosis to begin with. Regardless of the difficulty in discerning the exact cause of infection (whether from corticosteroids or due to an underdeveloped immune system), there is some correlation that this treatment can increase the risk of infection and complications. Therefore, it would be best to exercise caution; historically, this evidence has ultimately led to a reduction in the usage of postnatal corticosteroids.

\section{Neurodevelopmental outcomes}

A common adverse effect found in many studies which should be highlighted is impaired neurodevelopmental outcomes. ${ }^{42}$ An animal study found administration of corticosterone, another glucocorticoid, had adverse effects on the brain, including a reduction in brain size and impaired DNA synthesis, which may be involved in the mechanism underlying impaired neurodevelopment. ${ }^{43}$ This study also reported inhibited growth and a decrease in body weight as an adult. Another study on the effects of dexamethasone and betamethasone on the motor system, using neonatal rats as an animal model, reported negative effects on the central nervous system. ${ }^{44}$ One of the neurologic abnormalities presented was delayed development of vestibular reflexes (negative geotaxis response and free-fall righting response). However, the applicability of findings from animal models to humans is limited. The gestational period of rats is significantly shorter than that of humans (around 21 days). This can cause problems in translating equivalent stages of development because the order and timeline of development of organs and systems differ in rats and humans. These fac- 
tors are important because newborn rodents are often used as models for the preterm infant. The conclusions drawn from animal models should be critically assessed to determine the limitations when it is used as a model to understand effects that may occur in humans.

A number of trials have investigated the effects of postnatal corticosteroids on neurodevelopmental outcomes in humans. It is known that a potential complication of BPD is increased risk of impaired cognitive development. ${ }^{12}$ However, there are several reports showing that postnatal corticosteroid use may increase this pre-existing risk. ${ }^{35} \mathrm{~A}$ follow-up study on 190 infants in the Shinwell et al RCT reported an increase in the incidence of $\mathrm{CP}$ in those who were given dexamethasone. ${ }^{36}$ In this study, $49 \%$ of infants treated with dexamethasone presented with neurologic abnormalities such as spastic diplegia, a form of CP, whereas only $15 \%$ of those given placebo showed abnormalities. An observational study in 2010 concluded that the association between BPD and CP varies by the severity of the BPD and the $\mathrm{CP}$ phenotypes, when variables linked to prematurity are considered. ${ }^{45}$ However, this study has the potential limitations of all observational studies; results might be explained by failing to account for one or more unrecognized confounding factors.

Similar to rates of infection, it is difficult to determine how much of the observed neurologic outcomes are a result of corticosteroid treatment or a consequence of being born preterm. There are a range of possible confounding factors that may be involved in the outcomes seen. The preterm neonates in many of these trials have a poor prognosis; they often have a very low birth weight of $<1,500 \mathrm{~g}$ and are at high risk of other complications associated with being born preterm. Furthermore, although preterm birth is the largest risk factor for $\mathrm{CP}$, there are several others, including fetal growth restriction, infection, twin gestations and birth asphyxia. ${ }^{46}$ The trial and follow-up study by Shinwell et al does not give data on these risk factors that may have contributed to the development of $\mathrm{CP}{ }^{36}$ Thus, future trials that control for the risk factors for development of neurologic impairments should be investigated to quantify the contribution that postnatal corticosteroids may have in the incidence of neurologic impairment.

Despite these factors confounding the conclusions drawn from the trials, the frequency with which these impairments are reported strongly suggests that the use of postnatal corticosteroids, especially dexamethasone, does increase the risk of neurologic impairment.

\section{Long-term consequences}

There is a significant gap in the literature on the long-term consequences of perinatal corticosteroid treatment of the complications of prematurity. The widespread use of corticosteroids in neonatal care is relatively recent, becoming a common treatment after the initial trial by Liggins and Howie. ${ }^{19}$ As such, the earliest infants to receive prenatal or postnatal corticosteroids in the treatment and prevention of the complications of preterm birth have not yet reached old age. This lack of data is an important factor to consider when discussing the benefits and consequences of corticosteroid use; changes in body composition as we age can affect the sensitivity of steroid receptors. ${ }^{47}$ Thus, the long-term consequences on the physiological systems, such as the cardiovascular, respiratory and genitourinary systems, are not fully known. Furthermore, many infants who received postnatal corticosteroids were also exposed to them prenatally, which may influence postnatal treatment that is not yet known. Given the associations between high fetal glucocorticoid concentrations, low birth weight and adult disease, such as hypertension, there is the potential for unknown effects of postnatal corticosteroids to aggravate this long-term impact. Care needs to be taken with their use until we are fully aware of the long-term effects of postnatal corticosteroid treatment, as there may be consequences not yet observed.

\section{Alternatives to postnatal corticosteroids}

Given the various adverse physiological and neurodevelopmental effects that are associated with postnatal corticosteroid treatment, there have been several attempts to treat, mitigate or altogether eliminate them. These include alternative dosing and timing of regiments and even using different corticosteroids. Many of the trials on postnatal corticosteroids used dexamethasone. This has a longer half-life (36-72 hours) than other glucocorticoids, such as hydrocortisone (half-life of 8-12 hours) and may be more potent than the alternatives. ${ }^{48}$

\section{Alternative timings of administration}

NICUs often administer corticosteroids soon after birth if the infant is identified at risk of developing BPD. It is possible that the time at which they are administered may affect shortand long-term outcomes. A Cochrane review of 21 RCTs into the use of late postnatal corticosteroids in treating BPD was conducted with late defined as treatment in an infant older than 7 days of age. ${ }^{49}$ The review reported similar outcomes as those found in infants treated at an earlier postnatal age of $<7$ days. Late steroid treatment was associated with a reduction 
in neonatal mortality (at 28 days), but no reduction in mortality at 36 weeks. There was also a reduction in BPD both at 28 days of life and at 36 weeks PMA and a reduction in the need for assisted ventilation. However, short-term adverse effects included hyperglycemia, glycosuria and hypertension and an increase in the risk of infection and gastrointestinal bleeding. Thus, the authors concluded that late corticosteroid therapy may not outweigh actual or potential adverse effects, although it may reduce neonatal mortality without significantly increasing the risk of long-term neurodevelopmental outcomes (rates of death or CP were not significantly different between steroid and control groups).

\section{Alternative methods of administration}

Separate attempts have also been made to alter the method of administration to reduce the adverse effects of postnatal corticosteroids. An RCT compared the effects of postnatal corticosteroids administered via inhalation and intravenous injection. ${ }^{50}$ The authors hypothesized that the systemic effects could be reduced while maintaining the beneficial local effects. A total of 78 preterm infants on mechanical ventilation were entered into the trial. However, the authors found that although inhalation of corticosteroids resulted in similar improvements in lung function as intravenous administration, there was little difference in the adverse effects that it caused.

A notable limitation raised by the authors was that no control group was used, and the inhaled and intravenous groups were directly compared. The reason given was that steroids were the standard and common treatment for BPD at that time. This brings up ethical issues that may arise in the research of alternatives to established treatments, such as postnatal dexamethasone, in neonatal care and also in health care as a whole. The benefits of dexamethasone in improving the lung function and in earlier removal from mechanical ventilation are well supported and this has made it a standard treatment. When there are well-supported, standard treatments, it makes it difficult to justify and pursue alternative treatments (often with insufficient data on their efficacy and risks) that have the potential to be better than current ones. For this reason, obtaining more data and establishing control groups can be difficult when pursuing new treatments. This issue is apparent in prenatal corticosteroid treatment, where established treatments lead to inertia against novel drugs, dosing regimens and route of administration. However, the growing body of evidence on the negative effects of postnatal dexamethasone may be the impulse to remove this inertia, and may increase research to pursue alternative treatments to reduce the adverse effects.

\section{Alternative corticosteroids}

Less potent corticosteroids, such as hydrocortisone, may offer an opportunity to reduce the negative effects of dexamethasone. A study on neonatal rats found that dexamethasone was associated with impaired neurodevelopment, whereas hydrocortisone was not. ${ }^{51}$ The authors concluded that only neonatal dexamethasone altered the hippocampal synaptic plasticity and associative memory formation in later life and, thus, suggested that hydrocortisone may be a safer alternative. Furthermore, a retrospective study at two NICUs was carried out to compare the adverse effects and neurodevelopmental outcomes of neonates given postnatal dexamethasone or hydrocortisone. ${ }^{52}$ A tapering course of either $5-1 \mathrm{mg} / \mathrm{kg}$ hydrocortisone over 22 days or $0.5-0.1 \mathrm{mg} / \mathrm{kg}$ dexamethasone over 21 days was given to preterm infants. Control groups were also matched for gestational age, birth weight, severity of infant respiratory distress syndrome and period of admission. Despite the fact that the dose of hydrocortisone was 10 times higher than that of dexamethasone, the authors hypothesized that the longer biological half-life, lower glucocorticoid activity and higher mineralocorticoid activity of hydrocortisone may mitigate the negative effects compared to dexamethasone. The results showed that hydrocortisone was equally as effective as dexamethasone in improving the lung function and reducing the need for mechanical ventilation. The study also showed that hydrocortisone had less severe or absent side effects compared to dexamethasone. Hydrocortisone-treated infants showed same weight gain as the controls, whereas the dexamethasone-treated infants had significantly reduced weight gain compared to controls. A follow-up study on some of the infants at 5-7 years of age showed that the neurologic outcomes of the hydrocortisone-treated infants were better than those treated with dexamethasone. The dexamethasone group also had worse school performance compared to the control group, and a greater percentage of infants required special education. However, not all the infants in the initial study participated in the follow-up, and the relatively small number of infants assessed reduces the quality of the investigation on the long-term effects of hydrocortisone and its comparison to dexamethasone.

More recently, an RCT of 21 NICUs assessed whether low-dose hydrocortisone improved survival without BPD in extremely preterm infants. ${ }^{32}$ Infants randomly assigned to the hydrocortisone group received $1 \mathrm{mg} / \mathrm{kg}$ of hydrocortisone per day. This was divided into two doses per day for 7 days, followed by one dose of $0.5 \mathrm{mg} / \mathrm{kg}$ per day for 3 days. The authors found that low doses of prophylactic hydrocortisone increased survival without BPD at 36 weeks equivalent 
gestational age. Additionally, hydrocortisone treatment increased the proportion of patients extubated at the end of the study period, reduced the frequency of surgical ligation for PDA and increased the proportion of infants not requiring supplemental oxygen at 36 weeks of PMA. In the 26-27 week gestational age group, hydrocortisone also significantly reduced neonatal death before discharge. These data suggest that hydrocortisone may be a safer alternative in the treatment of BPD than dexamethasone.

\section{Preventive measures}

The adverse effects of postnatal corticosteroids can be avoided altogether by preventive measures to reduce the incidence of BPD. Mechanical ventilation is a common cause of BPD, and therefore, this should be addressed with safer methods and procedures; reducing the need for intubation could help to reduce the lung damage caused by barotrauma. Less-invasive methods for ventilation may be able to reduce the incidence of BPD and use of corticosteroids. Early nasal continuous positive airway pressure (NCPAP) is a noninvasive method of ventilation that is often used to avoid the need for intubation and the associated risks. Meta-analysis of NCPAP is also frequently carried out in conjunction with exogenous surfactant. Early surfactant therapy and early extubation, followed by NCPAP, is associated with a lower incidence of BPD compared with continued mechanical ventilation and later surfactant therapy. ${ }^{5}$ Thus, less-invasive and milder methods of ventilation may reduce barotrauma and the damage that it causes on the structurally immature and sensitive lungs of preterm infants, reducing the incidence of BPD.

\section{Conclusion}

With the use of both prenatal and postnatal corticosteroids to treat BPD, a careful balance must be obtained between the short-term benefits and the long-term consequences. Prenatal corticosteroids have been successful in reducing NRDS and neonatal mortality, but evidence suggests they have had little to no effect on the incidence rate of BPD. They may also have negative long-term effects due to developmental programming. On the other hand, postnatal corticosteroids have data that associate them with reduction in the severity of some cases of BPD, but not with decreased neonatal mortality. There are many studies that demonstrate their effectiveness in improving the lung function and earlier removal from mechanical ventilation. However, the short-term adverse effects associated with their use, such as hypertension, gastrointestinal bleeding and abnormal cardiac development, can be dangerous. Furthermore, the association between postnatal corticosteroid administration and neurodevelopmental impairment is a cause for concern.

One way to balance the benefits and negative effects is to restrict the use of postnatal corticosteroids to infants with severe BPD. This may be especially desirable in infants who are dependent on invasive mechanical ventilation as part of the standard treatment for NRDS. The use of corticosteroids in treating BPD should be considered on a case-by-case basis and alternatives should be used wherever possible to avoid the adverse effects. Furthermore, the lack of data on the long-term effects of perinatal corticosteroids presents a significant potential risk which should be investigated. Research should also be directed at finding methods that can better predict which infants are at greatest risk of preterm birth and BPD. More attention can then be given to women at risk of a premature delivery and preventive strategies can be taken. Furthermore, most infant deaths due to prematurity occur in developing countries, where using corticosteroids is not a standard practice. ${ }^{53}$ Cost-effective strategies to deploy treatments and preventive measures in these countries may make a significant contribution to reducing neonatal mortality due to BPD and prematurity worldwide.

\section{Author contributions}

$\mathrm{RM}$ and $\mathrm{OO}$ were involved in the conception, design, drafting, revising and final approval of the article. UO was involved in the design, drafting and final approval. All authors contributed toward data analysis, drafting and revising the paper and agree to be accountable for all aspects of the work.

\section{Disclosure}

The authors report no conflicts of interest in this work.

\section{References}

1. Blencowe H, Cousens S, Oestergaard MZ, et al. National, regional, and worldwide estimates of preterm birth rates in the year 2010 with time trends since 1990 for selected countries: a systematic analysis and implications. Lancet. 2012;379(9832):2162-2172.

2. Goldenberg RL, Culhane JF, Iams JD, Romero R. Epidemiology and causes of preterm birth. Lancet. 2008;371(9606):75-84.

3. Roberts D, Brown J, Medley N, Dalziel SR. Antenatal corticosteroids for accelerating fetal lung maturation for women at risk of preterm birth. Cochrane Database Syst Rev. 2017;3:CD004454.

4. Ramanathan R. Surfactant therapy in preterm infants with respiratory distress syndrome and in near-term or term newborns with acute RDS. $J$ Perinatol. 2006;26(Suppl 1):S51-S56.

5. Stevens TP, Harrington EW, Blennow M, Soll RF. Early surfactant administration with brief ventilation vs. selective surfactant and continued mechanical ventilation for preterm infants with or at risk for respiratory distress syndrome. Cochrane Database Syst Rev. 2007;4:CD003063. 
6. Northway WH Jr, Rosan RC, Porter DY. Pulmonary disease following respirator therapy of hyaline-membrane disease. Bronchopulmonary dysplasia. N Engl J Med. 1967;276(7):357-368.

7. Bancalari E, Abdenour G, Feller R, Gannon J. Bronchopulmonary dysplasia: clinical presentation. J Pediatr. 1979;95(5 Pt 2):819-823.

8. Shennan AT, Dunn MS, Ohlsson A, Lennox K, Hoskins EM. Abnormal pulmonary outcomes in premature infants: prediction from oxygen requirement in the neonatal period. Pediatrics. 1988;82(4):527-532.

9. Davidson LM, Berkelhamer SK. Bronchopulmonary dysplasia: chronic lung disease of infancy and long-term pulmonary outcomes. J Clin Med. 2017;6(12):4.

10. Ambalavanan N, Morty R. Searching for better animal models of BPD: a perspective. Am J Physiol Lung Cell Mol Physiol. 2016;311(5): L924-L927.

11. Berkelhamer SK, Mestan KK, Steinhorn RH. Pulmonary hypertension in bronchopulmonary dysplasia. Semin Perinatol. 2013;37(2):124-131.

12. Anderson PJ, Doyle LW. Neurodevelopmental outcome of bronchopulmonary dysplasia. Semin Perinatol. 2006;30(4):227-232.

13. Chess PR, D'Angio CT, Pryhuber GS, Maniscalco WM. Pathogenesis of bronchopulmonary dysplasia. Semin Perinatol. 2006;30(4):171-178.

14. Fowden AL, Li J, Forhead AJ. Glucocorticoids and the preparation for life after birth: are there long-term consequences of the life insurance? Proc Nutr Soc. 1998;57(1):113-122.

15. Padbury JF, Ervin MG, Polk DH. Extrapulmonary effects of antenatally administered steroids. J Pediatr. 1996;128(2):167-172.

16. Schellhase DE, Shannon JM. Effects of maternal dexamethasone on expression of SP-A, SP-B, and SP-C in the fetal rat lung. Am J Respir Cell Mol Biol. 1991;4(4):304-312.

17. Bolt RJ, van Weissenbruch MM, Lafeber HN, Delemarre-van de Waal HA. Glucocorticoids and lung development in the fetus and preterm infant. Pediatr Pulmonol. 2001;32(1):76-91.

18. Collins JJP, Tibboel D, de Kleer IM, Reiss IKM, Rottier RJ. The future of bronchopulmonary dysplasia: emerging pathophysiological concepts and potential new avenues of treatment. Front Med (Lausanne). 2017;4:61.

19. Liggins GC, Howie RN. A controlled trial of antepartum glucocorticoid treatment for prevention of the respiratory distress syndrome in premature infants. Pediatrics. 1972;50(4):515-525.

20. Kinsella JP, Greenough A, Abman SH. Bronchopulmonary dysplasia. Lancet. 2006;367(9520):1421-1431.

21. Tucker L, Hoff C, Peevy K, Brost B, Holland S, Calhoun B. The effects of antenatal steroid use in premature rupture of membranes. Aust NZ J Obstet Gynaecol. 1995;35(4):390-392.

22. Mulder EJ, Derks JB, Zonneveld MF, Bruinse HW, Visser GH. Transient reduction in fetal activity and heart rate variation after maternal betamethasone administration. Early Hum Dev. 1994;36(1):49-60.

23. Ehrenkranz RA. Single versus weekly courses of antenatal corticosteroids: evaluation of safety and efficacy. Yearbook Neonatal Perinatal Med. 2007;2007:25-26.

24. Reynolds RM. Glucocorticoid excess and the developmental origins of disease: two decades of testing the hypothesis - 2012 Curt Richter Award Winner. Psychoneuroendocrinology. 2013;38(1):1-11.

25. Kemp MW, Newnham JP, Challis JG, Jobe AH, Stock SJ. The clinical use of corticosteroids in pregnancy. Hum Reprod Update. 2016;22(2):240-259.

26. Clyman R. The role of patent ductus arteriosus and its treatments in the development of bronchopulmonary dysplasia. Semin Perinatol. 2013;37(2):102-107.

27. Chaudhary N, Filipov P, Bhutada A, Rastogi S. Controversies in the management of patent ductus arteriosus in preterm infants. $J$ Neonatal Biol. 2016;5:238.

28. Hong S, Levine L. Inhibition of arachidonic acid release from cells as the biochemical action of anti-inflammatory corticosteroids. Proc Natl Acad Sci USA. 1976;73(5):1730-1734.

29. Groneck P, Reuss D, Götze-Speer B, Speer CP. Effects of dexamethasone on chemotactic activity and inflammatory mediators in tracheobronchial aspirates of preterm infants at risk for chronic lung disease. J Pediatr. 1993;122(6):938-944.
30. Mammel M, Johnson D, Green T, Thompson T. Controlled trial of dexamethasone therapy in infants with bronchopulmonary dysplasia. Lancet. 1983;321(8338):1356-1358.

31. Jobe AH. Postnatal corticosteroids for bronchopulmonary dysplasia. Clin Perinatol. 2009;36(1):177-188.

32. Baud O, Maury L, Lebail F, et al. Effect of early low-dose hydrocortisone on survival without bronchopulmonary dysplasia in extremely preterm infants (PREMILOC): a double-blind, placebo-controlled, multicentre, randomised trial. Lancet. 2016;387(10030):1827-1836.

33. Walsh MC, Yao Q, Horbar JD, Carpenter JH, Lee SK, Ohlsson A. Changes in the use of postnatal steroids for bronchopulmonary dysplasia in 3 large neonatal networks. Pediatrics. 2006;118(5): e1328-e1335.

34. Stoll BJ, Hansen NI, Bell EF, et al. Trends in care practices, morbidity, and mortality of extremely preterm neonates, 1993 to 2012. Obstet Anesth Dig. 2016;36(2):76-77.

35. Doyle LW, Cheong JL, Ehrenkranz RA, Halliday HL. Early ( $<8$ days) systemic postnatal corticosteroids for prevention of bronchopulmonary dysplasia in preterm infants. Cochrane Database Syst Rev. 2017;10:CD001146.

36. Shinwell ES, Karplus M, Reich D, et al. Early postnatal dexamethasone treatment and increased incidence of cerebral palsy. Arch Dis Child Fetal Neonatal Ed. 2000;83(3):F177-F181.

37. Yeh TF. Prevention of chronic lung disease (CLD) in premature infants with early dexamethasone therapy. Pediatr Pulmonol Suppl. 1997;23(S16):35-36.

38. Zecca E, Papacci P, Maggio L, et al. Cardiac adverse effects of early dexamethasone treatment in preterm infants: a randomized clinical trial. J Clin Pharmacol. 2001;41(10):1075-1081.

39. Shah SS, Ohlsson A, Halliday HL, Shah VS. Inhaled versus systemic corticosteroids for preventing bronchopulmonary dysplasia in ventilated very low birth weight preterm neonates. Cochrane Database of Syst Rev. 2017;10:CD002058.

40. Shinwell ES, Karplus M, Zmora E, et al. Failure of early postnatal dexamethasone to prevent chronic lung disease in infants with respiratory distress syndrome. Arch Dis Child Fetal Neonatal Ed. 1996;74(1):F33-F37.

41. Durand M, Sardesai S, McEvoy C. Effects of early dexamethasone therapy on pulmonary mechanics and chronic lung disease in very low birth weight infants: a randomized, controlled trial. Pediatrics. 1995;95(6):584-590.

42. Barrington KJ. The adverse neuro-developmental effects of postnatal steroids in the preterm infant: a systematic review of RCTs. $B M C$ Pediatr. 2001;1:1.

43. Howard E. Reductions in size and total DNA of cerebrum and cerebellum in adult mice after corticosterone treatment in infancy. Exp Neurol. 1968;22(2):191-208.

44. Gramsbergen A, Mulder EJ. The influence of betamethasone and dexamethasone on motor development in young rats. Pediatr Res. 1998;44(1):105-110.

45. Van Marter LJ, Kuban KC, Allred E, et al; ELGAN Study Investigators. Does bronchopulmonary dysplasia contribute to the occurrence of cerebral palsy among infants born before 28 weeks of gestation? Arch Dis Child Fetal Neonatal Ed. 2011;96(1):F20-F29.

46. Stavsky M, Mor O, Mastrolia SA, Greenbaum S, Than NG, Erez O. Cerebral palsy-trends in epidemiology and recent development in prenatal mechanisms of disease, treatment, and prevention. Front Pediatr. 2017;5:21.

47. Hess GD, Joseph JA, Roth GS. Effect of age on sensitivity to pain and brain opiate receptors. Neurobiol Aging. 1981;2(1):49-55.

48. Gupta S, Prasanth K, Chen CM, Yeh TF. Postnatal corticosteroids for prevention and treatment of chronic lung disease in the preterm newborn. Int J Pediatr. 2012;2012:315642

49. Doyle LW, Cheong JL, Ehrenkranz RA, Halliday HL. Late (> 7 days) systemic postnatal corticosteroids for prevention of bronchopulmonary dysplasia in preterm infants. Cochrane Database Syst Rev. 2017;10:CD001145. 
50. Suchomski SJ, Cummings JJ. A randomized trial of inhaled versus intravenous steroids in ventilator-dependent preterm infants. J Perinatol. 2002;22(3):196-203.

51. Huang CC, Lin HR, Liang YC, Hsu KS. Effects of neonatal corticosteroid treatment on hippocampal synaptic function. Pediatr Res. 2007;62(3):267-270.
52. van der Heide-Jalving M, Kamphuis PJ, van der Laan MJ, et al. Short- and long-term effects of neonatal glucocorticoid therapy: is hydrocortisone an alternative to dexamethasone? Acta Paediatr. 2007;92(7):827-835.

53. Mwansa-Kambafwile J, Cousens S, Hansen T, Lawn JE. Antenatal steroids in preterm labour for the prevention of neonatal deaths due to complications of preterm birth. Int J Epidemiol. 2010;39(Suppl 1):i122-i133.

\section{Publish your work in this journal}

The International Journal of General Medicine is an international, peer-reviewed open-access journal that focuses on general and internal medicine, pathogenesis, epidemiology, diagnosis, monitoring and treatment protocols. The journal is characterized by the rapid reporting of reviews, original research and clinical studies across all disease areas.
The manuscript management system is completely online and includes a very quick and fair peer-review system, which is all easy to use. Visit http://www.dovepress.com/testimonials.php to read real quotes from published authors.

Submit your manuscript here: https://www.dovepress.com/international-journal-of-general-medicine-journal 